\title{
L'ANNEAU DE COLLISIONS A ÉLECTRONS ET POSITRONS D'ORSAY
}

\author{
P. MARIN \\ Groupe de l'anneau de collisions d'Orsay $\left(^{+}\right)$ \\ Laboratoire de l'Accélérateur Linéaire, Orsay
}

Une description très brève de la machine sera tout d'abord donnée : éléments magnétiques, système

$\left.{ }^{+}\right)$Ce groupe comprend, sous la direction du Professeur A. Blanc-Lapierre :

(1) Les physiciens et ingénieurs du Laboratoire de l'Accélérateur Linéaire d'Orsay et du Centre d'Etudes Nucléaires de Saclay qui ont participé au projet et à la construction d'ACO ainsi qu'aux essais et dont les noms suivent :

MM. G. Arzelier, J. E. Augustin, R. A. Beck, R. Belbéoch, M. Bergher, H. Bruck, G. Gendreau, P. Gratreau, J. Haissinski, R. Jolivot, G. Leleux, P. Marin, B. Milman, F. Rumpf, E. Sommer, H. Zyngier.

(2) Les physiciens qui ont participé à la réalisation du dispositif expérimental auprès d'ACO : MM. J. E. Augustin, J. Buon, J. Haissinski, F. Laplanche, P. Marin, F. Rumpf, E. Silva. d'injection, cavité R. F., chambres à vide, dispositifs d'observation des faisceaux, section expérimentale.

On insistera ensuite sur les performances attendues : gamme d'énergie utile, intensités stockées, luminosité, durée de vie des faisceaux, ainsi que sur quelques phénomènes limitant les valeurs de ces paramètres.

Les résultats de machine intéressant les expériences de physique, obtenus avec $\mathrm{ACO}$, seront ensuite résumés : rendement d'injection en électrons et positrons, durée de remplissage, durées de vie et dimensions des faisceaux, etc... On insistera tout particulièrement sur les problèmes de bruit de fond et de normalisation des sections efficaces. On discutera finalement les expériences en cours de préparation.

\section{LES ANNEAUX DE STOCKAGE A PROTONS DU CERN}

\author{
B. DE RAAD
}

Division ISR, CERN, Genève

L'ensemble des anneaux de stockage se compose de deux anneaux concentriques, qui se croisent en 8 points. L'énergie maximum des protons dans chaque anneau est de $28 \mathrm{GeV}$. Avec un courant de $20 \AA$ il y aura environ $1,5 \times 10^{5}$ collisions proton-proton par seconde à chaque intersection. La largeur et la hauteur $\mathrm{du}$ faisceau seront de $6 \mathrm{~cm}$ et $1 \mathrm{~cm}$ environ. La bande de quantité de mouvement est de $\Delta p / p= \pm 1 \%$

Dans les anneaux de stockage l'équipement d'expérimentation est très étroitement lié aux composants de la machine elle-même et le choix des paramètres de la machine peut donc avoir une grande influence sur les possibilités d'expérimentation. Nous avons choisi un angle de croisement de $15^{\circ}$. La longueur des sections droites aux points d'intersection est d'environ $16 \mathrm{~m}$. Il y aura deux halles d'expérimentation de conception assez différentes autour de deux points de croisement, tandis qu'à deux autres points le tunnel de la machine a été élargi jusqu'à $18 \mathrm{~m}$. Au-dessous des régions d'interaction il y a des grands puits dont le sol est à $5 \mathrm{~m}$ ou $3,8 \mathrm{~m}$ au-dessous du faisceau afin de permettre l'installation de grands appareils de détection tout autour des points de croisement. 